\title{
Metformin, A Potential Role in Age-Related Macular Degeneration: A Systematic Review and Meta-Analysis
}

\author{
Dewi Fathin Romdhoniyyah (D) - Simon P. Harding • \\ Christopher P. Cheyne · Nicholas A. V. Beare
}

Received: March 1, 2021 / Accepted: March 27, 2021 / Published online: April 12, 2021

(C) The Author(s) 2021

\begin{abstract}
Background: Currently, no generally approved medical treatment can delay the onset of agerelated macular degeneration (AMD) or slow the progression of degenerative changes. Repurposing drugs with beneficial effects on AMD pathophysiology offers a route to new treatments which is faster, cost-effective, and safer for patients. Recent studies indicate a potential role for metformin in delaying AMD development and progression. In this context, we conducted a systematic review and meta-analysis to look for beneficial associations between metformin and AMD.
\end{abstract}

Supplementary Information The online version contains supplementary material available at https:// doi.org/10.1007/s40123-021-00344-3.

D. F. Romdhoniyyah $(\bowtie) \cdot$ S. P. Harding ·

N. A. V. Beare

Department of Eye and Vision Science, Institute of Life Course and Medical Sciences, University of Liverpool, Liverpool L7 8TX, UK

e-mail: d.f.romdhoniyyah@liverpool.ac.uk

S. P. Harding $\cdot$ N. A. V. Beare

St. Paul's Eye Unit, Liverpool University Hospitals NHS Foundation Trust, Member of Liverpool Health Partners, Liverpool, UK

C. P. Cheyne

Department of Health Data Science, Institute of Population Health, University of Liverpool, Liverpool, UK
Methods: We systematically searched Medline and Embase (via Ovid), Web of Science, and ClinicalTrials.gov databases for clinical studies in humans that examined the associations between metformin treatment and AMD published from inception to February 2021. We calculated pooled odds ratio (OR) with 95\% confidence interval $(\mathrm{CI})$ considering a random effect model in the meta-analysis.

Results: Five retrospective studies met the inclusion criteria. There are no prospective studies that have reported the effect of metformin in AMD. The meta-analysis showed that people taking metformin were less likely to have AMD although statistical significance was not met (pooled adjusted $\mathrm{OR}=0.80,95 \%$ CI $\left.0.54-1.05, I^{2}=98.8 \%\right)$. Subgroup analysis of the association between metformin and early and late AMD could not be performed since the data was not available from the included studies.

Conclusions: Analysis of retrospective data suggests a signal that metformin may be associated with decreased risk of any AMD. It should be interpreted with caution because of the failure to meet statistical significance, the small number of studies, and the limitation of routine record data. However prospective studies are warranted in generalizable populations without diabetes, of varied ethnicities, and AMD stages. Clinical trials are needed to determine if metformin has efficacy in treating early and latestage AMD. 
Keywords: Metformin; Age-related macular degeneration; Age-related maculopathy; Systematic review; Meta-analysis

\section{Key Summary Points}

Metformin is postulated to delay ageing and ageing-related diseases, including agerelated macular degeneration.

We performed a systematic review and meta-analysis on five retrospective populations studies investigating metformin use and presence of AMD, showing an odds ratio of 0.80 (95\% CI 0.54-1.05)

Laboratory and clinical studies support a potential role for metformin in delaying AMD development and progression through multiple modes of action.

There are no published data from prospective studies of the effect of metformin on AMD. Prospective studies are needed to further investigate this positive signal.

\section{DIGITAL FEATURES}

This article is published with digital features, including a summary slide, to facilitate understanding of the article. To view digital features for this article go to https://doi.org/10.6084/ m9.figshare.14308706

\section{INTRODUCTION}

Age-related macular degeneration (AMD) is the most common cause of blindness in high-income countries with prevalence rising exponentially with age [1]. Globally, AMD is a top five cause of vision loss [2]. In the next decades with aging population, the number of people with AMD in the world is estimated to increase by $50 \%$ to 288 million in 2040 with the highest burden in Asia [1]. AMD can be divided into early and advanced stages [3] with the latter having serious impacts on vision. Advanced AMD has two forms: (1) atrophic/dry/non-neovascular and (2) neovascular/wet/exudative [4].

Treatments for AMD are limited to arresting neovascular AMD (nAMD). Anti-vascular endothelial growth factor (anti-VEGF) therapies, delivered by intravitreal injections, are successful at treating the active neovascular component of nAMD [5]. However, treatment is not curative, the benefit does not extend to atrophic or fibrotic changes, and requires a series of expensive injections over a long period of time [6]. Unfortunately, there is currently no therapy for geographic atrophy (GA) or other forms of degenerative AMD which in all account for approximately $90 \%$ of the disease burden [7]. Despite widespread opinion on antioxidant supplementation, the evidence of their benefit is weak [8-10]. The Age-Related Eye Disease Study (AREDS) [11] did not meet primary endpoints, and evidence of effect was based on post hoc subgroup analysis [12, 13]. Age-Related Eye Disease Study 2 (AREDS2) did not provide data on whether antioxidants are effective in preventing AMD progression as it did not include a placebo arm $[14,15]$.

Currently, dry or non-neovascular AMD remains an unmet medical need, and the commonest cause of registered blindness in highincome countries [16]. GA is the target of clinical trials for new treatments, but patients with non-GA, enlarging drusen, drusenoid pigment epithelial detachment (PED), and vitelliformtype degeneration are currently underserved in drug development pipelines. New therapies that are effective in the prevention of AMD and limiting its progression are urgently needed, since its incidence is increasing and because of limitations on existing therapies. Developing new drugs is expensive in cost and time [17]. Repurposing existing drugs to find new functional benefits or indications can be an alternative. There is growing interest in metformin as a candidate drug for treating AMD and reducing its progression. This is because of its antioxidant, anti-inflammatory, antiangiogenic, and antifibrotic effects [18-21]. Metformin is postulated to delay ageing and ageing- 
related diseases [22, 23]. Metformin is well tolerated and has a good safety profile with a long history as a treatment for type 2 diabetes mellitus (T2DM) [24]. Therefore, we performed a systematic review and conducted a meta-analysis underpinning the potential use of metformin in AMD.

\section{METHOD}

\section{Eligibility Criteria and Literature Search Strategy}

We performed a systematic review and metaanalysis following the guidelines of the Preferred Reporting Items for Systematic Reviews and Meta-Analyses (PRISMA) statement [25] and the Strengthening the Reporting of Observational studies in Epidemiology (STROBE) statement [26]. This article is based on previously conducted studies and does not contain any new studies with human participants or animals performed by any of the authors. Studies were included if they met the following criteria: (1) studies in human; (2) one study objective is to explore the association between metformin treatment and the risk of AMD; (3) there is control group; (4) the studies provided the effect estimate or calculable information from the available data presented in the article; (5) peer reviewed article; (6) the studies stated the definition and classification of AMD clearly in the paper; (7) published in English. The latest information published was to be used if there were serial publications using the same subjects.

A systematic electronic search of Medline and Embase (via Ovid) and Web of Science Databases including conference papers and abstracts from the earliest record in databases until February 2021 was performed. An additional search was performed on ClinicalTrials.gov to capture unpublished studies. The search strategy included the generic keyword combinations of "metformin" and "macular degeneration" with further details listed in Supplementary Material A. The references of eligible studies were also searched, and expert reference suggestion was included to make sure all relevant materials were captured.

\section{Study Selection}

We used Covidence software (Veritas Health Innovation, https://www.covidence.org) for data extraction, data organisation, and literature screening. After the duplicate check, two reviewers independently identified relevant titles and abstracts. Full-text articles were reviewed if on the basis of the title and abstract they appeared to meet the inclusion criteria, or if there was any uncertainty.

\section{Data Collection and Study Quality Assessment}

Data obtained included data source, sample size, study design, study period, AMD diagnostic criteria, the unadjusted and adjusted effect estimate and/or calculable information from the available data, adjustments for potential confounders, and study outcome. Quality of study assessment was conducted using the Downs and Black checklist [27, 28], which includes 27 items to assess the quality of reporting (10 items), external validity (3 items), internal validity including bias and confounding (13 items), and statistical power (1 item).

The original Downs and Black checklist allows a cumulative score of 32 points, one item having a maximum two points in the quality of reporting section and five points in the statistical power section. An adjustment was made to the scoring of item 27 that refers to the statistical power of the study. We rated a single point if the study had sufficient power to detect a clinically important effect, instead of scoring based on the available range of study powers. Therefore, the maximum score for the checklist was 28. The Downs and Black score ranges were assigned according to quality levels into four groups: less than 14 (low), 14-18 (moderate), 19-23 (good), and more than 23 (excellent) [29]. The quality of each study was independently assessed by two reviewers, with discrepancies resolved through discussion. 


\section{Data Synthesis and Statistical Analysis}

We converted any kind of risk estimates and any calculable information reported from the included studies to an odds ratio (OR) for pooled result calculation. Heterogeneity between studies was tested by Cochrane chisquare $\chi^{2}$ (Cochran $Q$ ) and $I^{2}$ statistic. The $P$ value less than 0.1 was set as a threshold for statistical significance in using Cochran's $Q$ statistical test to assess heterogeneity [30]. Threshold interpretation of heterogeneity in $I^{2}$ statistic was considered as low, moderate, and high for $I^{2}$ upper limits of $25 \%, 50 \%$, and $75 \%$, respectively [31]. A sensitivity analysis was conducted by removing single studies to determine if any individual study has a significant impact on the pooled OR. All analyses were conducted using STATA software version 16.1 (StataCorp LLC, Texas, USA).

\section{RESULTS}

\section{Literature Systematic Search}

A total of 204 records were screened from the literature search. Figure 1 shows the study selection process for articles included in metaanalysis. There was no disagreement between the two independent reviewers who conducted the study selection. We found five published retrospective studies using AMD diagnosis criteria based on the International Classification of Diseases Ninth and/or Tenth Edition (ICD-9, ICD-10) listed in Supplementary Material B with metformin medication documented from the medical records examining the relationship between AMD and the use of metformin medications (Table 1). Table 2 provides the methodological quality assessment of each included study according to the Downs and Black tool. All included studies were of moderate quality with scores ranging from 15 to 17 points, out of a possible 28 points.

Three studies were conducted in the USA. The first study [32] analysed 7788 patient records (1947 AMD and 5841 non-AMD) of people aged 55 years and older from the University of Florida electronic health record who attended at least four times from June 2011 to June 2017, and found that metformin was associated with substantially decreased risk of developing any AMD (ICD-9 diagnostic codes) using univariate analysis (OR $0.39 ; 95 \%$ CI 0.31-0.49, $P<0.001)$ and conditional multivariable logistic regression (OR 0.58; 95\% CI $0.43-0.79, P<0.001)$. In a subgroup analysis just of patients with diabetes, metformin use had decreased odds of developing any AMD (OR 0.70 ; 95\% CI $0.49-0.98, P=0.043)$. This study suggests a large reduction in risk of any AMD associated with metformin use, but is limited by its retrospective use of routine health records. Causality of course is not demonstrated since other inevitable exposure cannot be controlled in a non-randomised study and possibility of data misclassification.

The second study in the USA [33] gathered data from University of California San Francisco electronic medical record of people aged 60 years and older with diabetes, and having an ophthalmology encounter from April 2012 to August 2019. It reported that in 3120 patients, metformin was associated with decreased odds of any AMD (OR 0.70; 95\% CI 0.55-0.88, $P=0.003$ ) and non-neovascular AMD (OR 0.59; 95\% CI $0.46-0.77, P<0.001)$ using propensity score-weighted logistic regression models. It should be noted that this study only included patients who had seen an ophthalmologist, and this is more likely if they have AMD. This is a potential source of bias.

The third study in the USA [34] used data of 624,780 patient records $(312,404$ AMD and 312,376 non-AMD) of people aged 55 years and older who had eye examinations at least two times during the previous 12 months from IBM MarketScan Commercial and Medicare Supplemental Databases from January 2008 to December 2017. In multivariable logistic regression analysis, the use of metformin was reported to slightly lower the odds of having any AMD in a whole study population (OR 0.94; 95\% CI 0.92-0.96, $P<0.001)$ and in a subgroup of people with diabetes (OR 0.95; 95\% CI 0.93-0.97, $P<0.001$ ). The metformin doses of 1-270 g over 2 years had the highest reduction in odds of having AMD (OR 0.91; 95\% CI $0.89-0.94, P<0.001)$ compared to higher doses 


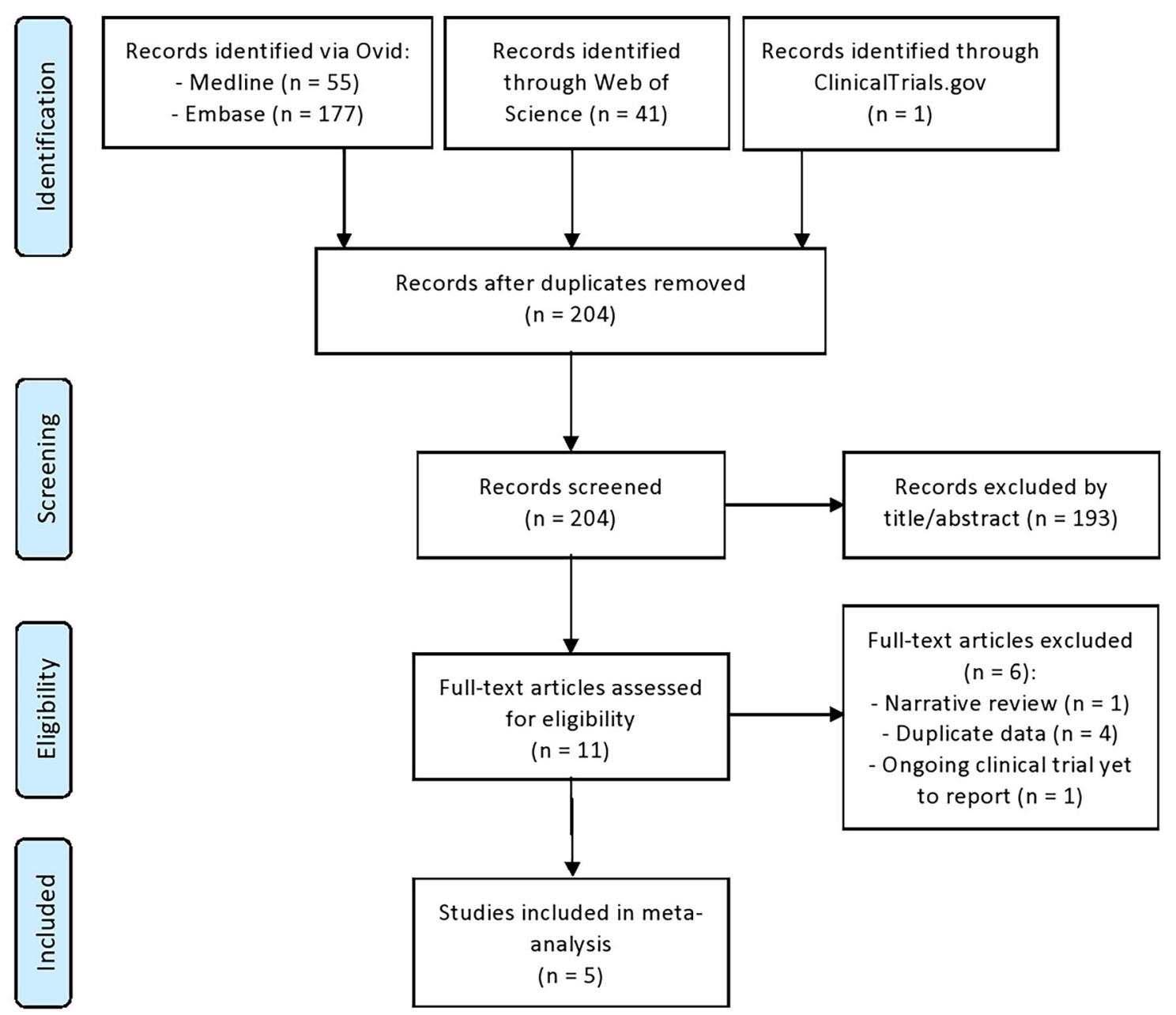

Fig. 1 PRISMA flowchart of study selection process

in a whole study population. This study matched the risk factors and comorbidities within 1 year before the index date, while metformin exposure was within 2 years before the index date. This introduced a risk of bias in sample selection since the comorbidities may have developed after metformin exposure.

A study in Taiwan [35] was a populationbased retrospective cohort study in patients with T2DM $(45,524$ patients taking metformin and 22,681 control subjects) using the Taiwan National Health Insurance Research Database from 2001 to 2013. Cox regression analysis demonstrated substantially less AMD developing within the metformin group after adjusting for age, sex, and systemic comorbidities compared to controls (HR 0.54; 95\% CI 0.50-0.58,
$P<0.001)$. People in the metformin group were still at a lower risk of AMD after adjustment of propensity score in the Cox regression analysis (HR $0.57 ; \quad 95 \%$ CI $0.52-0.63, \quad P<0.001$ ). Decreasing risk of AMD was observed with a longer duration and higher dose of metformin (Cox regression analysis, $P<0.05$ ).

A study in Korea using National Health Insurance Service data [36] included 2330 cases of AMD and 23,278 controls from patient records of people who were diagnosed with diabetes or cardiovascular diseases in 2002. Metformin users had no reduction in any AMD (ICD-10 diagnostic code) risk (adjusted OR 1.15, 95\% CI 0.91-1.45). The study concluded that metformin was not associated with a decreased risk of any AMD after adjusting for 


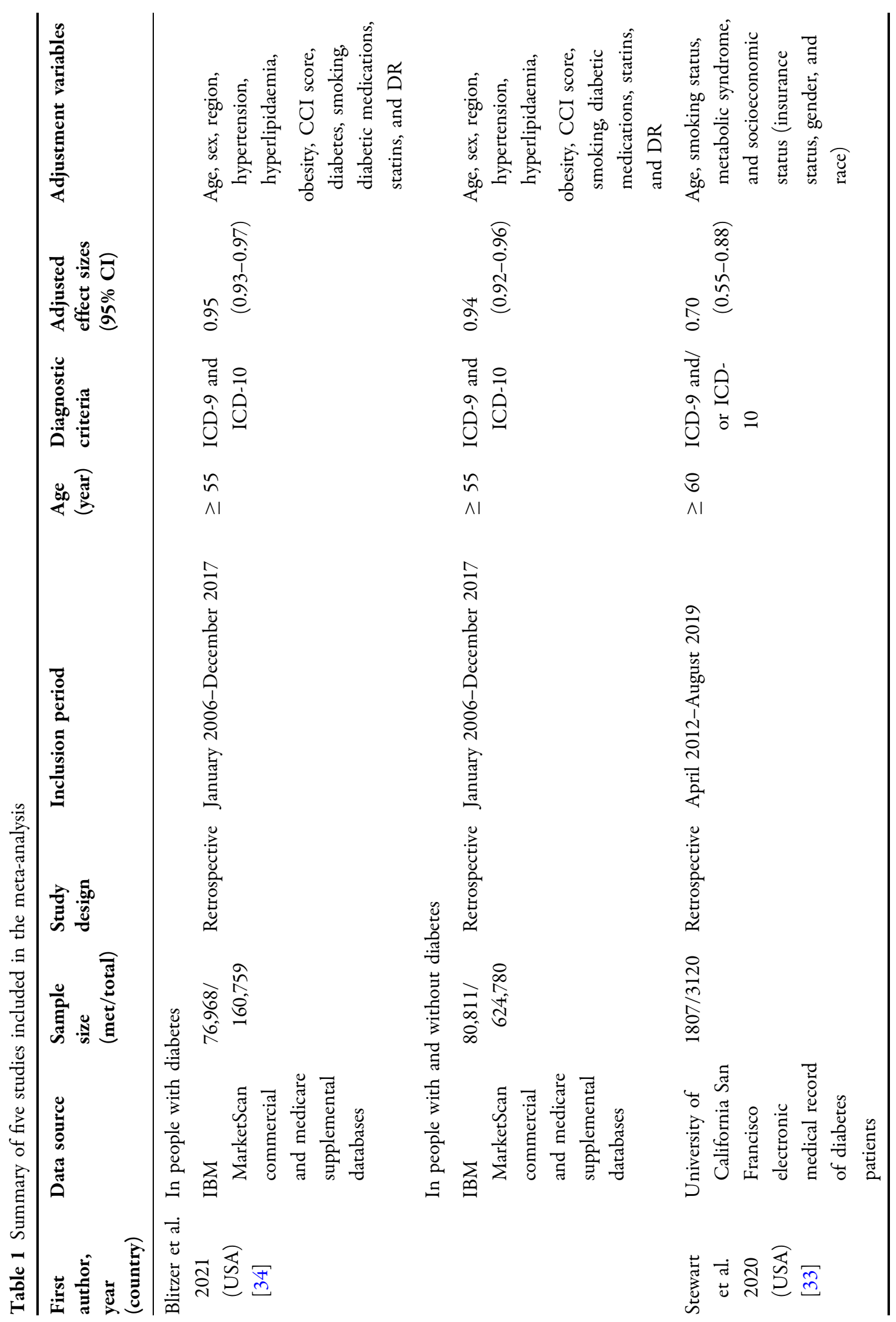




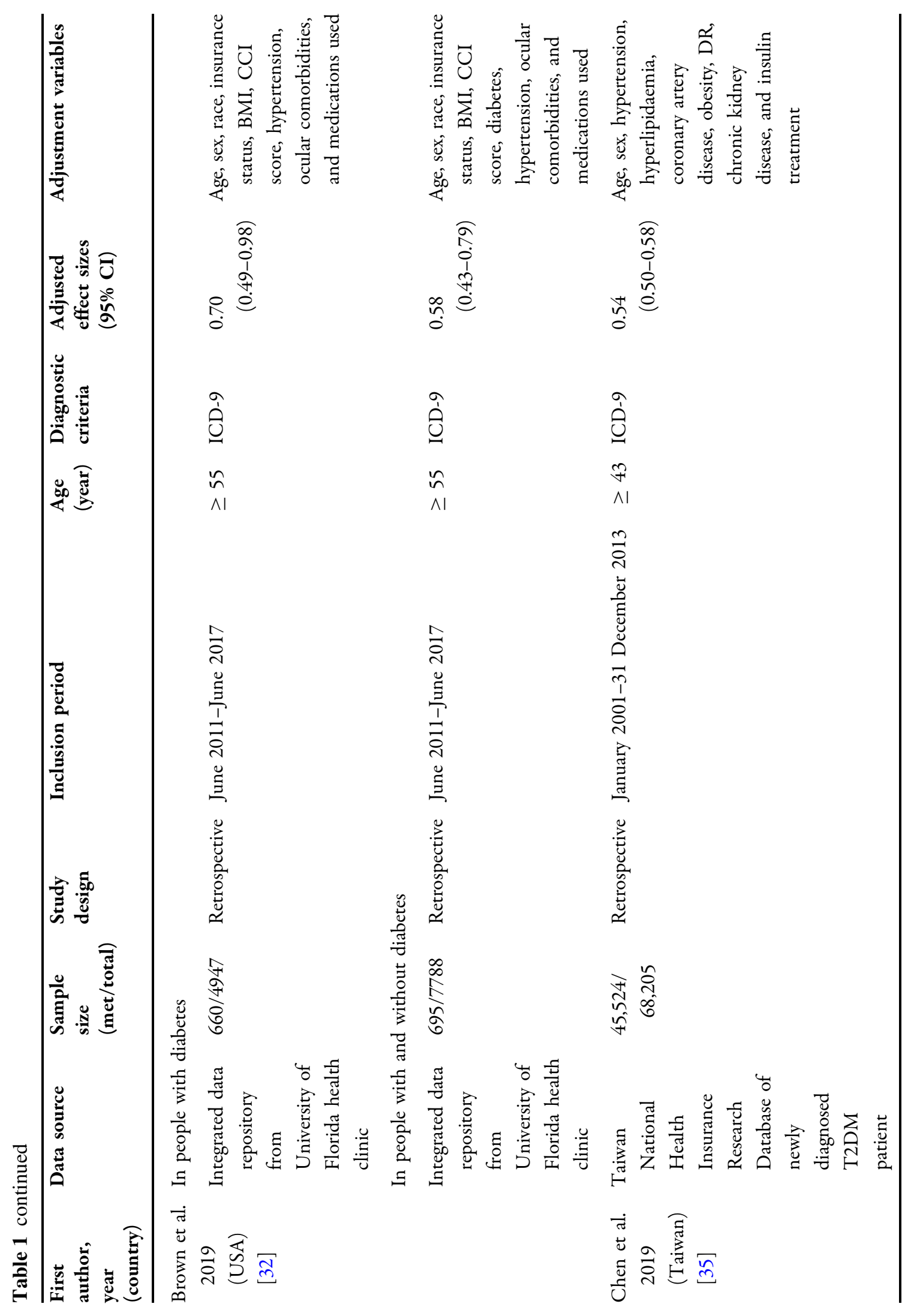




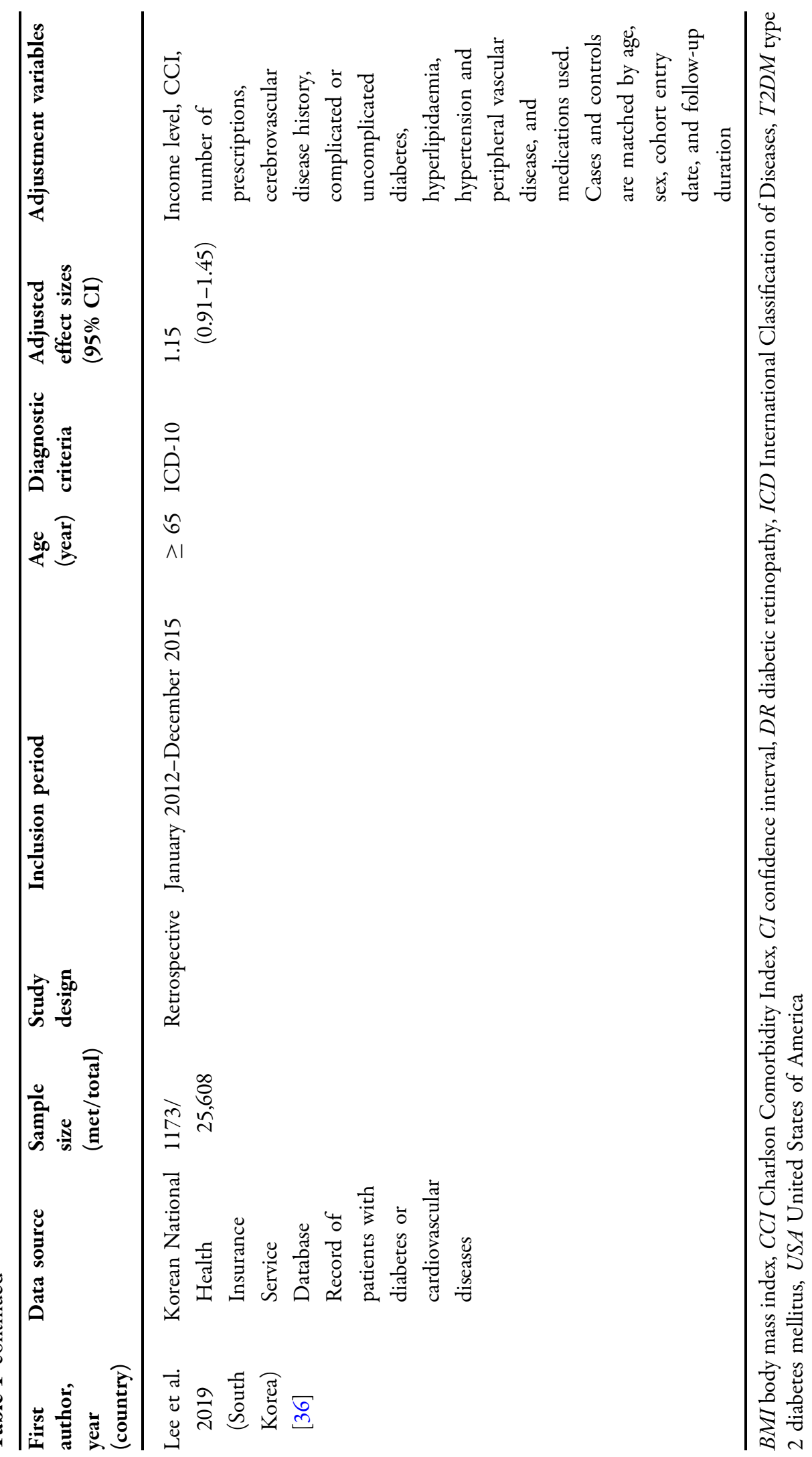


Table 2 Results of study quality assessment using the Downs and Black assessment tool

\begin{tabular}{|c|c|c|c|c|c|c|}
\hline \multirow[t]{2}{*}{ Study ID } & \multirow[t]{2}{*}{ Reporting } & \multirow[t]{2}{*}{ External validity } & \multicolumn{2}{|c|}{ Internal validity } & \multirow[t]{2}{*}{ Power } & \multirow[t]{2}{*}{ Total } \\
\hline & & & Bias & Confounding & & \\
\hline Blitzer et al. [34] & 9 & 1 & 3 & 3 & 1 & 17 \\
\hline Stewart et al. [33] & 8 & 1 & 2 & 3 & 1 & 15 \\
\hline Brown et al. [32] & 9 & 1 & 3 & 3 & 1 & 17 \\
\hline Chen et al. [35] & 9 & 1 & 3 & 3 & 1 & 17 \\
\hline Lee et al. [36] & 9 & 1 & 3 & 3 & 1 & 17 \\
\hline
\end{tabular}

socioeconomic status, healthcare resource utilization, combined medication use, and comorbidities. Compared to other studies, only this study included people with cardiovascular diseases as the study population. This is a potential source of bias as cardiovascular disease is reported to have an association with AMD [37-40].

We identified that there are no published prospective studies that have investigated metformin in AMD. Only two studies in the metaanalysis presented the association of metformin and AMD in people with and without diabetes $[32,34]$ and one study in people with diabetes or cardiovascular disease [36]. A phase 2 randomized clinical trial study began in 2016 to investigate the effects of metformin on the progression of geographic atrophy and drusen in AMD in patients without diabetes initiated by University of California; no data or results have been reported (ClinicalTrials.gov Identifier NCT02684578). The clinical trials completion date is expected in October 2021.

\section{META-ANALYSIS}

We performed a meta-analysis for the association of metformin and any AMD in people with diabetes. We could not generalize the results in people without diabetes, as only two studies reported data in this group [32, 34]. We analysed 'any AMD' since all studies included all AMD, not only atrophic and neovascular AMD, but also unspecified form of macular degeneration (Supplementary Material B). Only one study presented data of an AMD subtype, non- neovascular AMD, in the analysis [33]. Thus, it is not possible to perform meta-analysis by classifying the AMD subtypes.

The forest plot of our meta-analysis from the adjusted effect estimates of five retrospective studies is shown in Fig. 2. The overall OR of any AMD from metformin use was 0.80 with $95 \%$ CI 0.54-1.05. In a sensitivity analysis, the OR remained stable and the upper limit of the confidence interval remained close to 1.0, affecting the statistical significance (removing study by Blitzer et al. [34]: OR 0.75, 95\% CI 0.52-0.97, $P<0.001, I^{2}=87.1 \%$; Stewart et al. [33]: OR 0.82, 95\% CI 0.53-1.12, $P<0.001$, $I^{2}=99.1 \%$; Brown et al. [32]: OR 0.82, 95\% CI $0.54-1.11, P<0.001, I^{2}=99.1 \%$; Chen et al. [35]: OR 0.87, 95\% CI 0.70-1.05, $P=0.002$, $I^{2}=79.7 \%$; Lee et al. [36]: 0.72, 95\% CI 0.44-1.01, $\left.P<0.001, I^{2}=99.1 \%\right)$.

The heterogeneity of included studies was high $\left(I^{2}=98.8 \%\right)$. After excluding study by Chen et al. [35] and Blitzer et al. [34], we observed that the heterogeneity $I^{2}$ decreased from $98.8 \%$ to $79.7 \%$ and $87.1 \%$. We did not perform stratified analysis across a number of participants characteristic because of the small number of studies included.

\section{DISCUSSION}

In this novel systematic review and meta-analysis, we found a beneficial odds ratio between metformin use and decreased risk of AMD from retrospective data, although it is not statistically significant. Retrospective, routinely collected data will not determine whether metformin will 


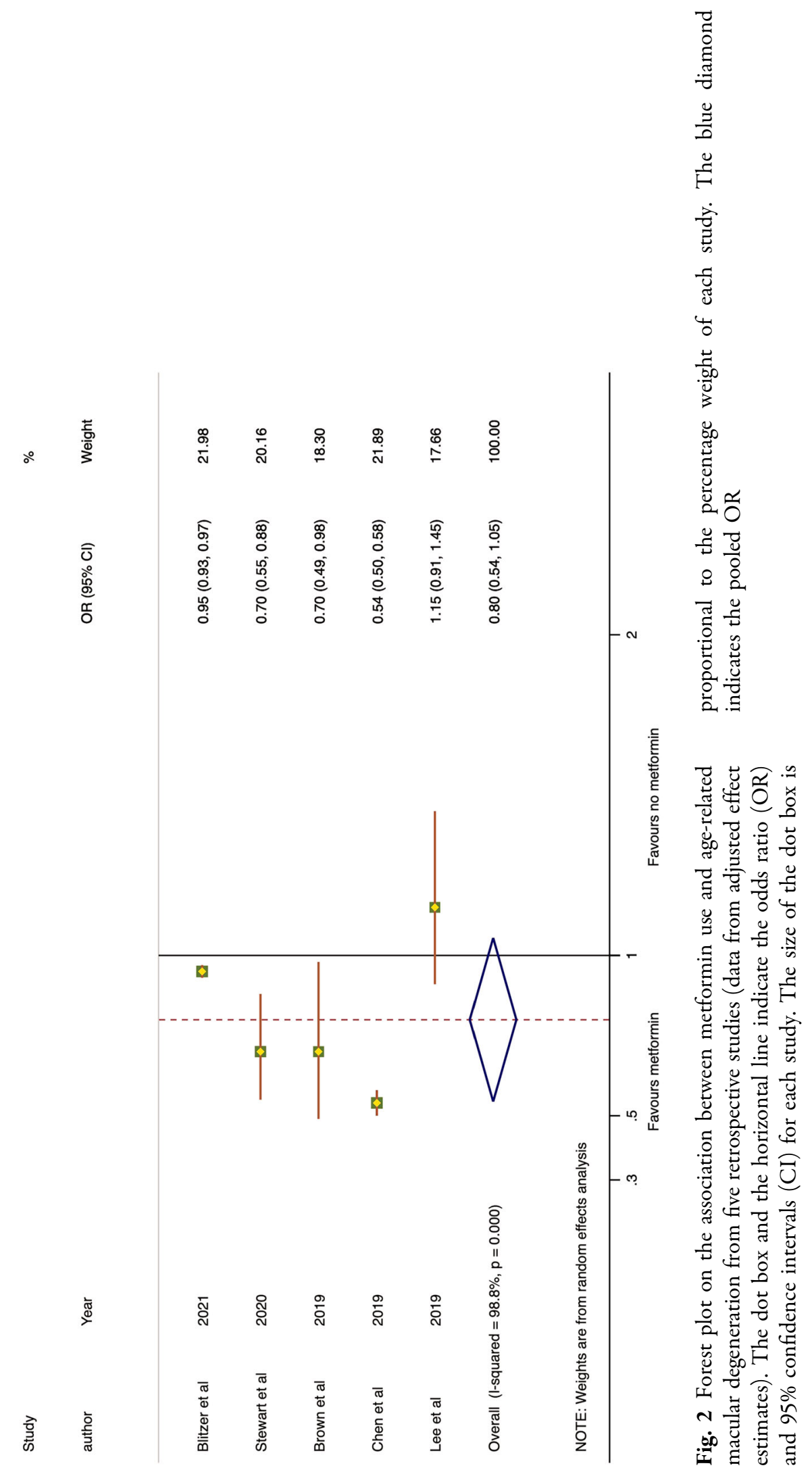


be of benefit for AMD, but the signal found justifies further research and clinical investigation. AMD is by far the commonest cause of blindness in wealthy countries with currently no treatment for the majority.

In the epidemiological studies, people with diabetes have shown a greater burden of AMD [41-43]. The pathophysiology of AMD is thought to share some pathways with diabetes, especially oxidative stress and chronic inflammation [41, 42, 44-48]. Laboratory and clinical studies have investigated the potential role of metformin in suppression of oxidative stress [49] and inflammation [50], supporting the hypothesis of its potential role in delaying AMD progression.

Metformin is considered to have a beneficial role in ageing-related diseases owing to its antioxidant [51-55] and anti-inflammatory effects [50]. Metformin suppresses oxidative stress on retinal pigment epithelium (RPE) by stimulating the AMPK (adenosine monophosphate-activated protein kinase) signalling pathway in an AMD mouse model [20]. The model uses sodium iodate to cause oxidative damage to RPE that imitates the oxidative stress in early AMD. AMPK is a cellular mechanism that is activated in response to metabolic stress conditions to restore energy homeostasis through balancing the adenosine triphosphate (ATP) levels [56]. ATP is vital to meet the high metabolic demands of the retina and RPE $[57,58]$. Increased ATP levels are observed in the retinal degenerative mouse model treated with metformin [20].

Ageing and oxidative stress are not only known for causing increased intracellular damage but are also thought to be related to impaired autophagy [59]. Metformin is able to induce autophagy and reduce apoptosis of retinal cells caused by vital dyes, indocyanine green (ICG) and brilliant blue G (BBG), in studies on human RPE cell lines (ARPE-19) and rat models [60]. This suggests the possible role of metformin in improving autophagy processes in AMD. Autophagy is important in cellular homeostasis for clearance of dysfunctional cell components [61]. A significant decrease in cellular autophagy is presumed to play an important role in development of AMD [62-64].
A systematic review and meta-analysis of metformin in neurodegenerative disease included 14 studies (seven cohorts, four cross-sectional, two RCTs, and one case-control) showed a protective effect against neurodegenerative diseases in patients with T2DM including dementia, Alzheimer's disease, and cognitive impairment in the elderly [22]. These findings in other diseases that associated with age and AMD [39-41, 65] promote a potential role for metformin delaying AMD and preventing its progression.

Abnormal angiogenesis driven by VEGF defines the neovascular form of AMD [66]. Metformin decreases levels of serum VEGF [67], so might have an effect on the macula angiogenic pathway. In mouse model and cultured human umbilical vein endothelial cell (HUVEC) studies of ischaemia-related retinopathy, metformin suppressed the vascular endothelial growth factor receptor (VEGFr) Flk-1, the key mediator of the angiogenic effects of VEGF [68]. Metformin is reported to reduce tumour progression with its antiangiogenic effect through suppression of hypoxic factors [69]. However in other studies, metformin has a cardioprotective effect by inducing angiogenesis, reducing angiogenic inhibitors, and increasing VEGFA [70]. The contradictory results of different studies on the angiogenic effects of metformin are thought to be due to tissue-specific angiogenic pathways $[19,71]$. Further research is needed to know the effect of metformin on angiogenesis in nAMD.

The transdifferentiation of cells to myofibroblasts or epithelial-mesenchymal transition (EMT) is related to subretinal fibrosis in AMD [72]. Suppression of EMT in RPE is reported to attenuate fibrosis [73], and metformin inhibits hypoxia-induced EMT in keloid fibroblasts [74]. Thus, EMT is a potential target of metformin in reducing fibrosis associated with late-stage AMD, warranting further research.

We have followed PRISMA and STROBE guidelines and using the adjusted effect estimates in the meta-analysis to ensure reliable results. However, some limitations need to be considered when interpreting our results. First, the literature search was limited to studies written in English, so there might be a language 
bias. Second, significant heterogeneity between studies exists, thereby limiting the strength of conclusions from this meta-analysis. Third, the population of included studies was based on US and East Asian populations, thus further studies in other locations and ethnicities are required. Our meta-analysis only included patients with diabetes as almost all patients routinely on metformin have diabetes; however prospective studies in non-diabetic populations are required to determine if an association exists in patients without diabetes. There is a first clinical trial investigating the effects of metformin on the progression of geographic atrophy and drusen in AMD in patients without diabetes (ClinicalTrials.gov Identifier NCT02684578). If this trial is positive, larger multicentre phase 2 and 3 studies will be needed to determine the clinical role of metformin in treating AMD.

Lastly, the studies included in our metaanalysis are retrospective, relatively few in number, and rely on data collected for other purposes. Therefore, the results must be interpreted cautiously. They use broad diagnostic categories of AMD so give no indication as to whether metformin may be beneficial in preventing progression of early AMD or improving outcomes in more advanced AMD. However, overall, they provide a signal of a possible beneficial effect of metformin in AMD. Although it is not statistically significant, further investigation into metformin's effect on AMD in prospective studies is warranted.

\section{CONCLUSION}

At present, data from five retrospective clinical studies include four with large reductions in risk of AMD associated with metformin. This is reflected in our meta-analysis albeit with an OR which does not meet significance at the 5\% level. Given metformin's potential favourable effects on AMD's pathophysiology, a reduction in risk seems plausible.

Prospective studies of metformin in AMD are needed to elucidate fully the role of metformin in AMD, as none reporting results were identified. The use of data and images from populations that are routinely screened for diabetic retinopathy also provides an opportunity to investigate metformin's effect on AMD cost-efficiently before conducting randomized clinical trials, since the data is collected prospectively and systematically. There is certainly an unmet treatment need at all stages of AMD which metformin has an exciting potential to fulfil. The mechanism of metformin as a treatment in AMD might due to its antioxidant, anti-inflammatory, antiangiogenic, and antifibrotic effects. Further study is needed to investigate whether these beneficial effects on RPE cells and in animal models are replicated in patients with AMD.

\section{ACKNOWLEDGEMENTS}

The authors wish to acknowledge Wega Wisesa Setiabudi, M.D. for taking the time as a second reviewer for the study selection and quality assessment.

Funding. The study and the journal's Rapid Service Fee were funded by The Ministry of Finance of Republic of Indonesia through Indonesia Endowment Fund for Education (Lembaga Pengelola Dana Pendidikan or LPDP) scholarship for doctoral study (grant number 201711220412052).

Authorship. All named authors meet the International Committee of Medical Journal Editors (ICMJE) criteria for authorship for this article, take responsibility for the integrity of the work as a whole, and have given their approval for this version to be published.

Author Contributions. Dewi Fathin Romdhoniyyah, Simon P Harding, Christopher P Cheyne, Nicholas AV Beare made a significant contribution to the work reported, including in the conception, study design, execution, acquisition of data, analysis and interpretation, or in all these areas; took part in drafting, revising or critically reviewing the article; gave final approval of the version to be published; have agreed on the journal to which the article 
has been submitted; and agree to be accountable for all aspects of the work.

Disclosures. Dewi Fathin Romdhoniyyah, Simon P Harding, Christopher P Cheyne, Nicholas AV Beare have nothing to disclose.

Compliance with Ethics Guidelines. This article is based on previously conducted studies and does not contain any new studies with human participants or animals performed by any of the authors.

Data Availability. Data sharing is not applicable to this article as no datasets were generated or analysed during the current study.

Open Access. This article is licensed under a Creative Commons Attribution-NonCommercial 4.0 International License, which permits any non-commercial use, sharing, adaptation, distribution and reproduction in any medium or format, as long as you give appropriate credit to the original author(s) and the source, provide a link to the Creative Commons licence, and indicate if changes were made. The images or other third party material in this article are included in the article's Creative Commons licence, unless indicated otherwise in a credit line to the material. If material is not included in the article's Creative Commons licence and your intended use is not permitted by statutory regulation or exceeds the permitted use, you will need to obtain permission directly from the copyright holder. To view a copy of this licence, visit http://creativecommons.org/licenses/by$\mathrm{nc} / 4.0 /$.

\section{REFERENCES}

1. Wong WL, Su X, Li X, et al. Global prevalence of age-related macular degeneration and disease burden projection for 2020 and 2040: a systematic review and meta-analysis. Lancet Glob Health. 2014;2:e106-16.

2. Flaxman SR, Bourne RRA, Resnikoff S, et al. Global causes of blindness and distance vision impairment 1990-2020: a systematic review and meta-analysis. Lancet Glob Health. 2017;5:e1221-34.
3. Ferris FL, Wilkinson CP, Bird A, et al. Clinical classification of age-related macular degeneration. Ophthalmology. 2013;120:844-51.

4. Hanus J, Zhao F, Wang S. Current therapeutic developments in atrophic age-related macular degeneration. Br J Ophthalmol. 2016;100:122-7.

5. Solomon SD, Lindsley K, Vedula SS, Krzystolik MG, Hawkins BS. Anti-vascular endothelial growth factor for neovascular age-related macular degeneration. Cochrane Database Syst Rev. 2014;8:005139.

6. Fernández-Robredo $\mathrm{P}$, Sancho A, Johnen $\mathrm{S}$, et al. Current treatment limitations in age-related macular degeneration and future approaches based on cell therapy and tissue engineering. J Ophthalmol. 2014;2014:510285.

7. Chiou GCY. Pharmacological treatment of dry agerelated macular degeneration (AMD). Taiwan J Ophthalmol. 2011;1:2-5.

8. Evans JR, Lawrenson JG. Antioxidant vitamin and mineral supplements for slowing the progression of age-related macular degeneration. Cochrane Database Syst Rev. 2017. https://doi.org/10.1002/ 14651858.CD000254.pub4/full/es.

9. Evans JR, Lawrenson JG. Antioxidant vitamin and mineral supplements for preventing age-related macular degeneration. Cochrane Database Syst Rev. 2017. https://doi.org/10.1002/14651858.CD000253.pu b4/full.

10. Waugh N, Loveman E, Colquitt J, et al. Treatments for dry age-related macular degeneration and Stargardt disease: a systematic review. Health Technol Assess. 2018;22:1-168.

11. AREDS. A randomized, placebo-controlled, clinical trial of high-dose supplementation with vitamins $C$ and $\mathrm{E}$, beta carotene, and zinc for age-related macular degeneration and vision loss: AREDS Report No. 8. Arch Ophthalmol. 2001;119:1417-36.

12. Ambati J, Ambati BK. Age-related eye disease study caveats. Arch Ophthalmol. 2002;120:997-997.

13. Seigel D. AREDS investigators distort findings. Arch Ophthalmol. 2002;120:100-100.

14 AREDS2. Lutein + zeaxanthin and omega-3 fatty acids for age-related macular degeneration: the AgeRelated Eye Disease Study 2 (AREDS2) randomized clinical trial. JAMA. 2013;309:2005-15.

15. Chew EY, Clemons T, SanGiovanni JP, et al. The age-related eye disease study 2 (AREDS2): study design and baseline characteristics (AREDS2 Report Number 1). Ophthalmology. 2012;119:2282-9. 
16. Rees A, Zekite A, Bunce C, Patel PJ. How many people in England and Wales are registered partially sighted or blind because of age-related macular degeneration? Eye. 2014;28:832-7.

17. Li YY, Jones SJ. Drug repositioning for personalized medicine. Genome Med. 2012;4:27.

18. Guan M, Li W, Xu L, et al. Metformin improves epithelial-to-mesenchymal transition induced by TGF- $\beta 1$ in renal tubular epithelial NRK-52E cells via inhibiting Egr-1. J Diabetes Res. 2018;2018: e1031367. https://doi.org/10.1155/2018/1031367.

19. Han J, Li Y, Liu X, et al. Metformin suppresses retinal angiogenesis and inflammation in vitro and in vivo. PLoS One. 2018;13:e0193031.

20. Xu L, Kong L, Wang J, Ash JD. Stimulation of AMPK prevents degeneration of photoreceptors and the retinal pigment epithelium. Proc Natl Acad Sci USA. 2018;115:10475-80.

21. Yi Q-Y, Deng G, Chen N, et al. Metformin inhibits development of diabetic retinopathy through inducing alternative splicing of VEGF-A. Am J Transl Res. 2016;8:3947-54.

22. Campbell JM, Stephenson MD, de Courten B, Chapman I, Bellman SM, Aromataris E. Metformin use associated with reduced risk of dementia in patients with diabetes: a systematic review and meta-analysis. J Alzheimers Dis. 2018;65:1225-36.

23. Kulkarni AS, Brutsaert EF, Anghel V, et al. Metformin regulates metabolic and nonmetabolic pathways in skeletal muscle and subcutaneous adipose tissues of older adults. Aging Cell. 2018;17(2):e12723.

24. Marshall SM. 60 years of metformin use: a glance at the past and a look to the future. Diabetologia. 2017;60:1561-5.

25. Moher D, Liberati A, Tetzlaff J, Altman DG. Preferred reporting items for systematic reviews and meta-analyses: the PRISMA statement. Int J Surg. 2010;8:336-41.

26. von Elm E, Altman DG, Egger M, Pocock SJ, Gøtzsche PC, Vandenbroucke JP. The strengthening the reporting of observational studies in epidemiology (STROBE) statement: guidelines for reporting observational studies. J Clin Epidemiol. 2008;61: 344-9.

27. Deeks JJ, Dinnes J, D'Amico R, et al. Evaluating non-randomised intervention studies. Health Technol Assess. 2003;7(27).

28. Downs $\mathrm{SH}$, Black N. The feasibility of creating a checklist for the assessment of the methodological quality both of randomised and non-randomised studies of health care interventions. J Epidemiol Commun Health. 1998;52:377-84.

29. O'Connor SR, Tully MA, Ryan B, Bradley JM, Baxter GD, McDonough SM. Failure of a numerical quality assessment scale to identify potential risk of bias in a systematic review: a comparison study. BMC Res Notes. 2015;8:224.

30. West SL, Gartlehner G, Mansfield AJ, et al. Summary of common statistical approaches to test for heterogeneity (Table 7). Comparative Effectiveness Review Methods: Clinical Heterogeneity. Agency for Healthcare Research and Quality. 2010. https:// www.ncbi.nlm.nih.gov/books/NBK53317/table/ ch3.t2/. Accessed 2020 Oct 7.

31. Higgins JPT, Thompson SG, Deeks JJ, Altman DG. Measuring inconsistency in meta-analyses. BMJ. 2003;327:557-60.

32. Brown EE, Ball JD, Chen Z, Khurshid GS, Prosperi $\mathrm{M}$, Ash JD. The common antidiabetic drug metformin reduces odds of developing age-related macular degeneration. Invest Ophthalmol Vis Sci. 2019;60:1470-7.

33. Stewart JM, Lamy R, Wu F, Keenan JD. Relationship between oral metformin use and age-related macular degeneration. Ophthalmol Retina. 2020;4(11): 1118-9.

34. Blitzer AL, Ham SA, Colby KA, Skondra D. Association of metformin use with age-related macular degeneration: a case-control study. JAMA Ophthalmol. 2021;1:1. https://doi.org/10.1001/jamao phthalmol.2020.6331.

35. Chen Y-Y, Shen Y-C, Lai Y-J, et al. Association between metformin and a lower risk of age-related macular degeneration in patients with type 2 diabetes. J Ophthalmol. 2019;2019:1649156.

36. Lee H, Jeon H-L, Park SJ, Shin J-Y. Effect of statins, metformin, angiotensin-converting enzyme inhibitors, and angiotensin II receptor blockers on agerelated macular degeneration. Yonsei Med J. 2019;60:679-86.

37. Chakravarthy U, Wong TY, Fletcher A, et al. Clinical risk factors for age-related macular degeneration: a systematic review and meta-analysis. BMC Ophthalmol. 2010;10:31.

38. Cheung CMG, Wong TY. Is age-related macular degeneration a manifestation of systemic disease? New prospects for early intervention and treatment. J Intern Med. 2014;276:140-53.

39. Wang J, Xue Y, Thapa S, Wang L, Tang J, Ji K. Relation between age-related macular degeneration 
and cardiovascular events and mortality: a systematic review and meta-analysis. Biomed Res Int. 2016;2016:8212063.

40. Wu J, Uchino M, Sastry SM, Schaumberg DA. Agerelated macular degeneration and the incidence of cardiovascular disease: a systematic review and meta-analysis. PLoS One. 2014;9(3):e89600.

41. Chen X, Rong SS, Xu Q, et al. Diabetes mellitus and risk of age-related macular degeneration: a systematic review and meta-analysis. PLoS One. 2014;9(9): e108196.

42. He M-S, Chang F-L, Lin H-Z, Wu J-L, Hsieh T-C, Lee $\mathrm{Y}-\mathrm{C}$. The association between diabetes and age-related macular degeneration among the elderly in Taiwan. Diabetes Care. 2018;41:2202-11.

43. Vassilev ZP, Ruigómez A, Soriano-Gabarró M, Rodríguez LAG. Diabetes, cardiovascular morbidity, and risk of age-related macular degeneration in a primary care population. Invest Ophthalmol Vis Sci. 2015;56:1585-92.

44. Das UN. Diabetic macular edema, retinopathy and age-related macular degeneration as inflammatory conditions. Arch Med Sci. 2016;12:1142-57.

45. Evans JR. Risk factors for age-related macular degeneration. Prog Retin Eye Res. 2001;20:227-53.

46. Hahn P, Acquah K, Cousins SW, Lee PP, Sloan FA. Ten-year incidence of age-related macular degeneration according to diabetic retinopathy classification among medicare beneficiaries. Retina. 2013;33:911-9.

47. Hua R, Li Q, Wong IYH, Ning H, Wang H. Choroidal microvascular proliferation secondary to diabetes mellitus. Oncotarget Impact J. 2016;8:2034-6.

48. Rivera JC, Dabouz R, Noueihed B, Omri S, Tahiri H, Chemtob S. Ischemic retinopathies: oxidative stress and inflammation. Oxid Med Cell Longev. 2017;2017:3940241.

49. Esteghamati A, Eskandari D, Mirmiranpour H, et al. Effects of metformin on markers of oxidative stress and antioxidant reserve in patients with newly diagnosed type 2 diabetes: a randomized clinical trial. Clin Nutr. 2013;32:179-85.

50. Cameron AR, Morrison VL, Levin D, et al. Anti-inflammatory effects of metformin irrespective of diabetes status. Circ Res. 2016;119:652-65.

51. Barzilai N, Crandall JP, Kritchevsky SB, Espeland MA. Metformin as a tool to target aging. Cell Metab. 2016;23:1060-5.
52. Blagosklonny MV. An anti-aging drug today: from senescence-promoting genes to anti-aging pill. Drug Discov Today. 2007;12:218-24.

53. Chukwunonso Obi B, Chinwuba Okoye T, Okpashi VE, Nonye Igwe C, Olisah Alumanah E. Comparative study of the antioxidant effects of metformin, glibenclamide, and repaglinide in alloxan-induced diabetic rats. J Diabetes Res. 2016;2016:1635361.

54. Fang J, Yang J, Wu X, et al. Metformin alleviates human cellular aging by upregulating the endoplasmic reticulum glutathione peroxidase 7 . Aging Cell. 2018;17:e12765.

55. Hou X, Song J, Li X-N, et al. Metformin reduces intracellular reactive oxygen species levels by upregulating expression of the antioxidant thioredoxin via the AMPK-FOXO3 pathway. Biochem Biophys Res Commun. 2010;396:199-205.

56. Rabinovitch RC, Samborska B, Faubert B, et al. AMPK maintains cellular metabolic homeostasis through regulation of mitochondrial reactive oxygen species. Cell Rep. 2017;21:1-9.

57. Eells JT. Mitochondrial dysfunction in the aging retina. Biology (Basel). 2019;8(2):31.

58. Wong-Riley M. Energy metabolism of the visual system. Eye Brain. 2010;2:99-116.

59. Mitter SK, Rao HV, Qi X, et al. Autophagy in the retina: a potential role in age-related macular degeneration. In: LaVail MM, Ash JD, Anderson RE, Hollyfield JG, Grimm C, editors, et al. Retinal degenerative diseases. Boston: Springer; 2012. p. 83-90.

60. Shu C-W, Tsen C-L, Li M-S, Bee Y-S, Lin S-H, Sheu S-J. Metformin and rapamycin protect cells from vital dye-induced damage in retinal pigment epithelial cells and in vivo. Graefes Arch Clin Exp Ophthalmol. 2020;258:557-64.

61. Chun Y, Kim J. Autophagy: an essential degradation program for cellular homeostasis and life. Cells. 2018;7(12):278.

62 Golestaneh N, Chu Y, Xiao Y-Y, Stoleru GL, Theos AC. Dysfunctional autophagy in RPE, a contributing factor in age-related macular degeneration. Cell Death Dis. 2018;8:e2537-e2537.

63. Kaarniranta K, Tokarz $\mathrm{P}$, Koskela A, Paterno J, Blasiak J. Autophagy regulates death of retinal pigment epithelium cells in age-related macular degeneration. Cell Biol Toxicol. 2017;33:113-28.

64. Kivinen N. The role of autophagy in age-related macular degeneration. Acta Ophthalmol. 2018;96: $1-50$. 
65. Kaarniranta K, Salminen A, Haapasalo A, Soininen $\mathrm{H}$, Hiltunen $\mathrm{M}$. Age-related macular degeneration (AMD): Alzheimer's disease in the eye? J Alzheimers Dis. 2011;24:615-31.

66. Penn JS, Madan A, Caldwell RB, Bartoli M, Caldwell RW, Hartnett ME. Vascular endothelial growth factor in eye disease. Prog Retin Eye Res. 2008;27: 331-71.

67. Ersoy C, Kiyici S, Budak F, et al. The effect of metformin treatment on VEGF and PAI-1 levels in obese type 2 diabetic patients. Diabetes Res Clin Pract. 2008;81:56-60.

68. Joe SG, Yoon YH, Choi JA, Koh J-Y. Anti-angiogenic effect of metformin in mouse oxygen-induced retinopathy is mediated by reducing levels of the vascular endothelial growth factor receptor Flk-1. PLoS One. 2015;10:e0119708.

69. Wang J-C, Li G-Y, Li P-P, et al. Suppression of hypoxia-induced excessive angiogenesis by metformin via elevating tumor blood perfusion. Oncotarget. 2017;8:73892-904.
70. Bakhashab S, Ahmed FW, Schulten H-J, et al. Metformin improves the angiogenic potential of human CD34+ cells co-incident with downregulating CXCL10 and TIMP1 gene expression and increasing VEGFA under hyperglycemia and hypoxia within a therapeutic window for myocardial infarction. Cardiovasc Diabetol. 2016;15:27.

71. Dallaglio K, Bruno A, Cantelmo AR, et al. Paradoxic effects of metformin on endothelial cells and angiogenesis. Carcinogenesis. 2014;35:1055-66.

72. Shu DY, Lovicu FJ. Myofibroblast transdifferentiation: the dark force in ocular wound healing and fibrosis. Prog Retin Eye Res. 2017;60:44-65.

73. Hyttinen JMT, Kannan R, Felszeghy S, Niittykoski $\mathrm{M}$, Salminen A, Kaarniranta K. The regulation of NFE2L2 (NRF2) signalling and epithelial-to-mesenchymal transition in age-related macular degeneration pathology. Int J Mol Sci. 2019;20:5800.

74. Lei R, Zhang S, Wang Y, Dai S, Sun J, Zhu C. Metformin inhibits epithelial-to-mesenchymal transition of keloid fibroblasts via the HIF-1 $\alpha /$ PKM2 signaling pathway. Int J Med Sci. 2019;16:960-6. 